\title{
FEATURES OF IMPLEMENTATION OF THE MAXIMUM FORCES AND ACCELERATIONS IMPACT OF SOLIDS
}

Annotation. The problem of determining the maximum strength under shock interaction of solids in the case of elastic, plastic deformation of the contact surfaces, and when you hit the console with rectangular and circular cross-section. Determine the maximum strain in the center of the contact zone of the collision of bodies of the same and different materials. The quantitative relationship between the individual parameters in order to maximize the impact shock forces. An example of the practical application of the theoretical curves to create a shock copra high-speed interaction of the striker with the test object.

Keywords: shock interaction, high speed, elastic and plastic deformation, maximum force, pile driver for impact test.

\section{REFERENCES}

1.Tarasov V.N. Impact theory in theoretical mechanics and its application in the construction / V.N. Tarasov, GN Boyarkin.-Omsk: Publishing OmSTU, 1999.-120 with.

2. Galdin N.S. Optimization synthesis of the basic parameters of hydraulic pulse systems

construction machinery / N.S. Galdin, V.N. Galdin, NN Egorov // Herald SibADI.-2013.-№6 (34) .- S.73-77.

3. Shcherbakov V.S. Key indicators of hydraulic pulse systems of construction machinery of N.S. Shcherbakov, V.N. Galdin // Herald SibADI.-2013.-№1 (29).-S.47-51.

4. Alimov O.D. Hydraulic vibro-impact systems / O.D. Alimov, S.A. Basov. M .: Nauka, 1990.352s.

5. A.S 1045041 USSR, G 01 M 7 / 00. An apparatus for impact testing products [Text] B.N. Stickhanovskiy / -№3448940 / 25-28; zayavl.08.06.82; publ. 09.30.83, Bul.№36-3s.

6. Stickhanovskiy B.N. Shot process: Monograph / B.N. Stihanovsky .- Omsk: Publishing house OmSTU, 2010.324 with.
7. Pat. 163315 R.F. G 01 M 7/ 00 .Pile driver for impact test [Text] / B.N. Stickhanovskiy .E.S. Chernova; publ10.06.2016. Bul.№16.

Стихановский Борис Николаевич - д-р техн. наук, профрессор Омского государственного университета путей сообщения (644046, е. Омск, пр. Маркса, 35.,e-mail bstish@mail.ru.

Стихановская Любовь Михайловна - канд. техн. наук, доцент Сибирского государственного автомобильно-дорожного универcumema ( 644080, г.Омск ,пр. Mupa,5, e-mail stikhanovskaya@gmail.com.

Stickhanovskiy Boris Nikolaevich - d-r of technical Sciences, professor Omsk State Transport University (644046, Marksa,35 prospekt, Omsk, Russian Federation., e-mail bstish@mail.ru .

Stickhanovskaya Lubov Mihailovna candidate of technical Sciences, associate Professor Sibirskaya State Automobile and Highway University (644080, Mira,5 prospect, Omsk, Russian Federation., stikhanovskaya@ gmail.com.

\section{ВЛИЯНИЕ КООРДИНАТ ТОЧКИ КРЕПЛЕНИЯ УРАВНОВЕШИВАЮЩЕГО КАНАТА НА КОЛЕБАНИЯ ГРУЗА КРАНА-ТРУБОУКЛАДЧИКА}

В.В. Танский Сибирский государственный автомобильно-дорожный университет «СибАДИ», Россия, г. Омск

Аннотация: в работе рассмотрены вынужденные колебания груза на стреле крана-трубоукладчика, закрепленного при помощи уравновешивающего каната, обоснованна плоская расчетная схема крана-трубоукладчика и составлены диффреренциальные уравнения движения 
крана-трубоукладчика. Получены зависимости максимального отклонения груза от координат точки крепления уравновешивающего каната. Определено рациональное значение точки крепления уравновешивающего каната для каждого из исследуемых вылетов стрелы.

Ключевые слова: кран-трубоукладчик, колебания, уравновешивающий канат, груз.

\section{ВВЕДЕНИЕ}

С целью снижения амплитуды колебаний груза на стреле крана-трубоукладчика (КТ) предложено устройство закрепления груза на стреле КТ с помощью уравновешивающего каната, который существенно снижает колебания груза [3].

\section{ИССЛЕДОВАНИЕ КОЛЕБАНИЙ ГРУЗА}

Для исследования колебаний груза, закрепленного с помощью уравновешивающего каната, составлена расчетная схема рис. 1. На рис. изображены: 1 - базовый трактор, 2 стрела, 3 - грузовой канат, 4 - груз, 10 - уравновешивающий канат, 8,9 - опоры, упруго-вязкие свойства уравновешивающего, стрелового канатов и ходового оборудования учтены при помощи упруго - вязких тел Фохта 5,6 и 7 соответственно.

При составлении схемы были приняты следующие допущения:

1. КТ является стационарной и голономной системой.

2. Люфты и силы сухого трения в шарнирах отсутствуют $[2,4]$.

Для составления дифференциальных уравнений движения КТ необходимо задать системы координат [4].

Для описания положения всех звеньев си-

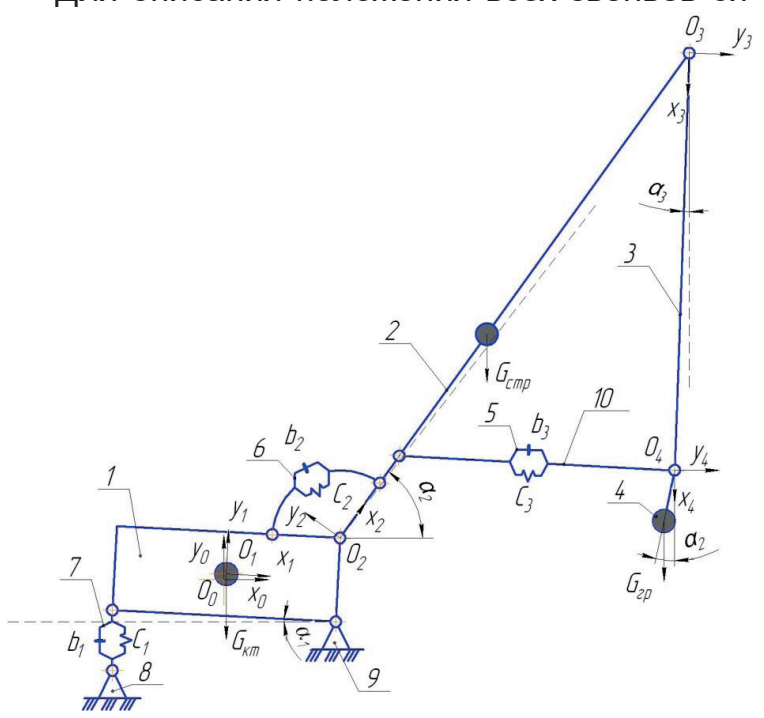

Рuc. 1. Расчетная схема крана-трубоукладчика стемы в двумерном пространстве был использован метод однородных координат. Данный метод значительно упрощает процесс определение координат и скоростей характерных точек при переходе из одной системы координат в другую, сводя его к одной операции матричного умножения [1, 2, 4].

Заданы следующие системы координат: инерциальная $\mathrm{O}_{0} \mathrm{X}_{0} \mathrm{Y}_{0}$ с началом в точке $\mathrm{O}_{0}$, совпадающим с центром масс первого звена в исходном положении и жестко связанная с неподвижным основанием. Для оценки угловых смещений отдельных звеньев системы были введены локальные системы координат связанные со звеньями. Угловое смещение базового трактора оценивается при помощи системы координат $\mathrm{O}_{1} \mathrm{X}_{1} \mathrm{Y}_{1}$ с началом в точке $\mathrm{O}_{1}$, совпадающим с центром масс первого звена. Угловое смещение стрелы - $\mathrm{O}_{2} \mathrm{X}_{2} \mathrm{Y}_{2}$ с началом в точке $\mathrm{O}_{2}$, совпадающим с шарниром крепления стрелы. Угловое смещение грузового каната - $\mathrm{O}_{3} \mathrm{X}_{3} \mathrm{Y}_{3}$ с началом в точке $\mathrm{O}_{3}$, совпадающим с шарниром крепления грузового каната. Угловое смещение груза $-\mathrm{O}_{4} \mathrm{X}_{4} \mathrm{Y}_{4}$ с началом в точке $\mathrm{O}_{4}$, совпадающим с шарниром крепления уравновешивающего каната.

Демпфирование воздействия на ходовое оборудование, а также демпфирования колебания груза и стрелы уравновешивающим и стреловым канатами было реализовано за счет упруго - вязких тел Фохта, которые в свою очередь характеризуются коэффицциентами жесткости $\mathrm{c}_{1}, \mathrm{c}_{2}, \mathrm{c}_{3}$ и вязкости $\mathrm{b}_{1}, \mathrm{~b}_{2}, \mathrm{~b}_{3}[2,4]$. Для каждого звена, имеющего массу, вычислены и заданы инерционные характеристики такие как: собственные моменты инерции $\mathrm{J}_{\mathrm{ix}}, \mathrm{J}_{\mathrm{iy}}$, и центробежные моменты инерции $\mathrm{J}_{\text {ixy, }}$ относительно локальных систем координат [2, 4].

Были введены следующие обобщенные координаты, обозначение которых представлено в табл. 1,

Для описания кинематики звеньев плоской расчетной схемы, согласно методу однородных координат [4, 5, 9], были составлены матрицы перехода из локальной системы координат в инерциальную, формулы (1-4):

$$
T_{1}=A_{x_{1}} \cdot A_{y_{1}} \cdot A_{\alpha_{1}}
$$




\section{СООТВЕТСТВИЕ МЕЖДУ УГЛОВЫМИ ПЕРЕМЕЩЕНИЯМИ И ОБОБЩЕННЫМИ КООРДИНАТАМИ}

\begin{tabular}{|l|c|}
\hline Перемещение & Обобщенная координата \\
\hline Поворот базового трактора на угол $\alpha_{1}$ & $\mathrm{q}_{1}$ \\
\hline Поворот стрелы в на угол $\alpha_{2}$ & $\mathrm{q}_{2}$ \\
\hline Поворот грузового каната на угол $\alpha_{3}$ & $\mathrm{q}_{3}$ \\
\hline Поворот груза на угол $\alpha_{4}$ & $\mathrm{q}_{4}$ \\
\hline
\end{tabular}

$$
\begin{aligned}
& T_{2}=A_{x_{2}} \cdot A_{y_{2}} \cdot A_{\alpha_{2}} \cdot T_{1} ; \\
& T_{3}=A_{x_{3}} \cdot A_{\alpha_{3}} \cdot T_{1} \cdot T_{2} ; \\
& T_{4}=\cdot A_{x_{4}} \cdot A_{\alpha_{4}} \cdot T_{1} \cdot T_{2} \cdot T_{3},
\end{aligned}
$$

где $\mathrm{A}_{\mathrm{ai}}$ - матрица поворота i-го звена на угол $\alpha_{\mathrm{i}}$, $A_{\mathrm{xi}}$-матрица параллельного переноса по оси $\mathrm{O}_{\mathrm{i}} \mathrm{X}_{\mathrm{i}}, \mathrm{A}_{\mathrm{yi}}$-матрица параллельного переноса по оси $\mathrm{O}_{\mathrm{i}} \mathrm{Y}_{\mathrm{i}}$.

Матрицы скорости U имеют следующий вид $[4,6]$ :

$$
\begin{aligned}
& U_{1}=A_{x_{1}} \cdot A_{y_{1}} \cdot E_{\alpha} \cdot A_{\alpha_{1}} ; \\
& U_{2}=A_{x_{2}} \cdot A_{y_{2}} \cdot E_{\alpha} \cdot A_{\alpha_{2}} \cdot T_{1} ; \\
& U_{3}=A_{x_{3}} \cdot E_{\alpha} A_{\alpha_{3}} \cdot T_{1} \cdot T_{2} ; \\
& U_{4}=A_{x_{4}} \cdot E_{\alpha} \cdot A_{\alpha_{4}} \cdot T_{1} \cdot T_{2} \cdot T_{3},
\end{aligned}
$$

где E - диффреренцирующая матрица по соответствующему углу $\alpha$.

Полученные матрицы позволяют определить положение и скорость характерных точек звеньев системы. Учитывая, что тело Фохта 1, принадлежит базовому трактору, тело 2 относится к стреле КТ, положение точек тела Фохта 3 описывается координатами в локальной системе координат грузового каната. Матрицы перехода Г и матрицы скоростей М будут иметь вид $[4,7,8]$ :

$$
\begin{aligned}
& \Gamma_{1}=A_{x_{1}} \cdot A_{y_{1}} \cdot A_{\alpha_{1}} \\
& \Gamma_{2}=A_{x_{2}} \cdot A_{y_{2}} \cdot A_{\alpha_{2}} \cdot T_{1} \\
& \Gamma_{3}=A_{x_{3}} \cdot A_{\alpha_{3}} \cdot T_{1} \cdot T_{2} \\
& M_{1}=U_{1} \\
& M_{2}=U_{2} \\
& M_{3}=U_{3} .
\end{aligned}
$$

Полученные матрицы позволяют определить положение и скорости подвижных концов упруго-вязких тел в произвольный момент времени $[2,4]$.

Полученный матрицы позволяют записать уравнение Лагранжа второго рода в векторно-матричной форме $[2,4]$ :

$$
\begin{aligned}
& \quad \sum_{i=1}^{4} \sum_{j=1}^{4} \operatorname{tr}\left[U_{i j} H_{i} U_{i v}^{T}\right] q_{j}^{\prime \prime}+ \\
& +\sum_{u=1}^{3} \sum_{j=1}^{4} \operatorname{tr}\left[M_{u j} B_{u} M_{u v}^{T}\right] q_{j}^{\prime}+ \\
& +\sum_{u=1}^{3} \sum_{j=1}^{4} \operatorname{tr}\left[M_{u j} N_{u} M_{u v}^{T}\right] q_{j}+ \\
& +\sum_{i=1}^{4} m_{i} g G^{T} U_{i v} \vec{R}_{i}=\vec{F}_{s} U_{i v} \vec{R}_{i r},
\end{aligned}
$$




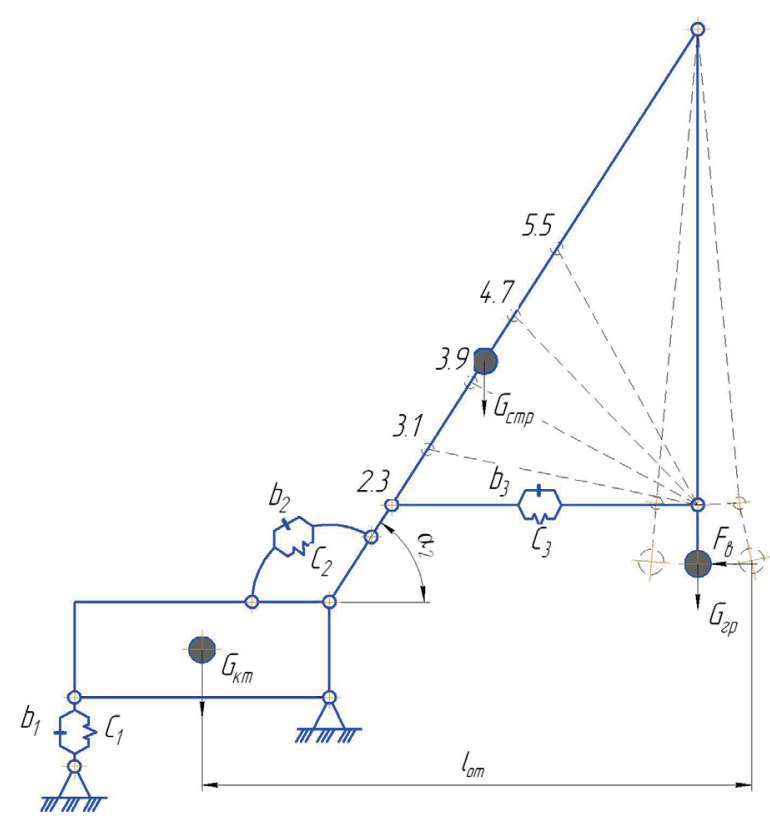

Puc. 2. Схема проведения эксперимента

где 4 - количество звеньев, 4 - количество степеней свободы, 3 - количество упругих элементов, $\bigcup_{\mathrm{ij}}$ - матрица скорости і-го элемента по ј-ой координате, $\mathrm{H}_{\mathrm{i}}$ - матрица инерционности і-го элемента, $\mathrm{M}_{\mathrm{uj}}$ - матрица деформации u-го упругого элемента по ј-ой координате, $\mathrm{N}_{\mathrm{u}}$ - матрица упругости, $B_{u}$ - матрица вязкости u-го упругого элемента, $\mathrm{G}^{\top}$ - вектор сил тяжести звеньев системы, $\vec{R}_{i}$ - радиус вектор і-го звена, $\mathrm{m}_{\mathrm{i}}$ - масса і-го звена, $M_{u v}^{T}$ - транспонированная матрица десрормации u - упругого элемента; $U_{i v}^{T}$ - транспонированная матрица скорости і-го элемента; $q_{j}$ - обобщенная координата по степени свободы j, $\mathrm{F}_{\text {в }}$ - сила, приложенная к і звену расчетной схемы; $\mathrm{R}_{\mathrm{ir}}$ - вектор координат точки приложения силы к звену і в локальной системе координат этого звена.

C целью получения зависимости максимального отклонения груза от состояния равновесия от координат точки крепления уравновешивающего каната был проведен машинный эксперимент. По результатам эксперимента определено рациональное значение точки крепления уравновешивающего каната для каждого из исследуемых вылетов стрелы, при фиксированном ступенчатом воздействии силы $\mathrm{F}_{\text {в. }}$.

На рис. 3 показана схема проведения эксперимента. К центру масс груза кратковремен- но прикладывалось ффиксированное значение силы $F_{\text {в }}$, что приводило к колебаниям и соответственно отклонению груза от состояния равновесия.

В процессе эксперимента измерялось максимальное отклонение груза І ${ }_{\text {от, }}$ с уравновешивающим канатом и без, как расстояние от центра масс груза до центра масс базового трактора и рассчитывалась разница $\Delta \mathrm{l}$ :

$$
\Delta l=l_{o m}^{0}-l_{o m}
$$

где $l_{\text {om }}^{0}$ - отклонение груза без уравновешивающего каната, $l_{\text {om }}$ - отклонение груза с уравновешивающим канатом.

После чего изменялся угол наклона стрелы $\alpha_{2}$ и снова рассчитывалась $\Delta$ l. По полученным данным была построена зависимость $\Delta \mathrm{l}=\mathrm{f}\left(\alpha_{2}\right)$. Данные зависимости были построены для различных точек крепления уравновешивающего каната, как показано на рис. 2.

\section{ЗАКЛЮЧЕНИЕ}

Наиболее предпочтительными координатами точек крепления уравновешивающего каната является 3,1 и 3,9 м от шарнира крепления стрелы, так как разница отклонений в данных случаях максимальна.

Из рис. 3 видно, что $\Delta \mathrm{I}$, характеризующая работу уравновешивающего каната, на разных точках крепления изменяется по разным законам. Такое отличие объясняется изменением угла наклона уравновешивающего каната к грузовому, и как следствие, разным значением угла наклона вектора уравновешивающей силы.

\section{БИБЛИОГРАФИЧЕСКИЙ СПИСОК}

1. Щербаков, В.С. Система автоматизации моделирования стреловых грузоподъемных кранов : монография / В.С. Щербаков, М.С. Корытов, С.В. Котькин. - Омск : СибАДИ, 2012. $-143 \mathrm{C}$.

2. Щербаков, В.С. Автоматизация процесса моделирования траектории движения рабочего органа робота-манипулятора : монография / В.С. Щербаков И.А. Реброва, М.С. Корытов, Е.И. Постухова. - Омск: Филиал ГОУ ВПО «Российский заочный институт текстильной и легкой промышленности» в г. Омске, 2009. 120c.

3. Пат. 158094 РФ: МПКВ66С23/26. кран-тру- 


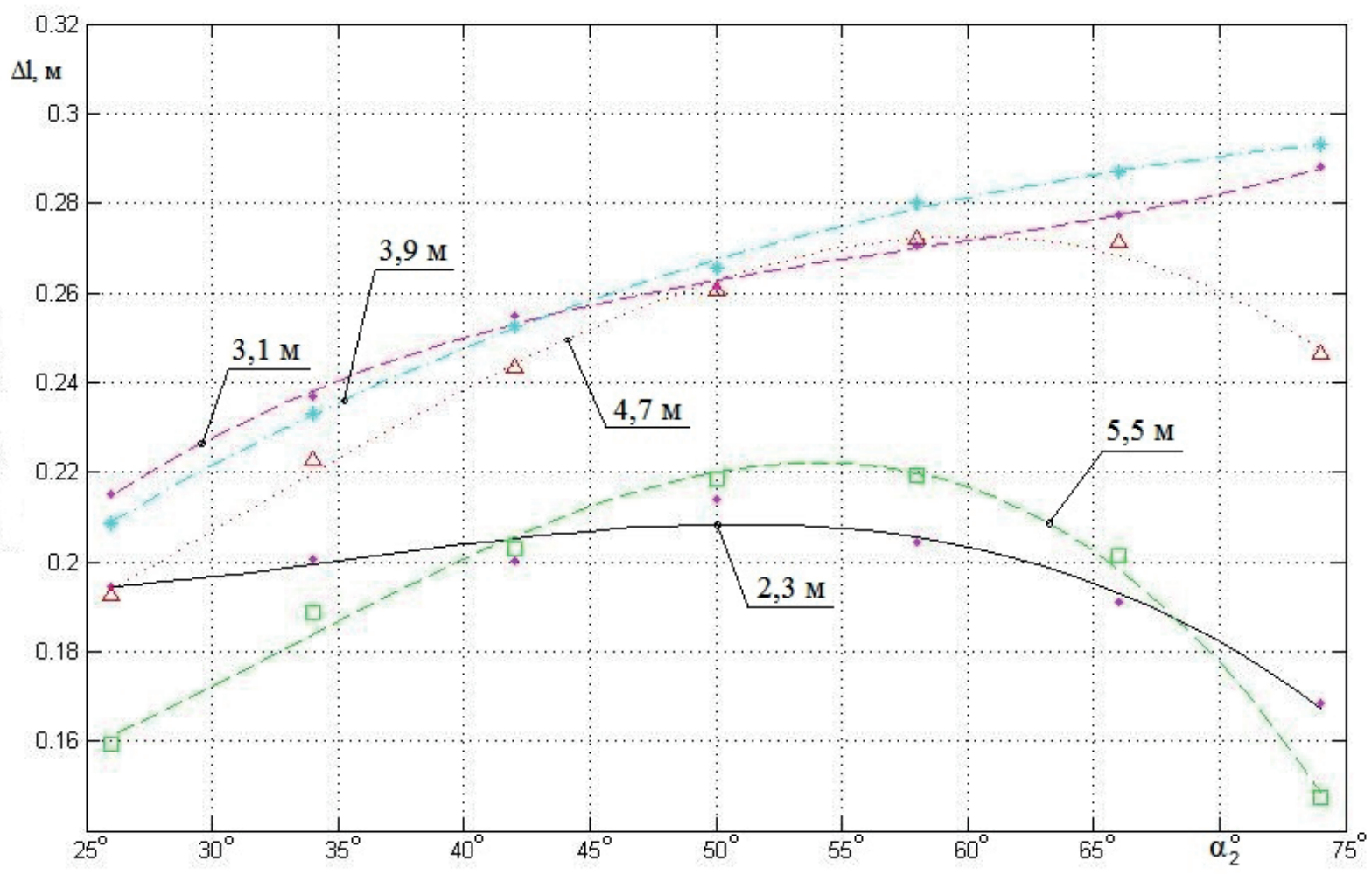

Puс. 3. Грасрики зависимостей разницы отклонений $\Delta /$ от угла наклона стрелы $\alpha_{2}^{\circ}$

боукладчик / Щербаков В.С., Корытов М.С., Танский В.В. ; СибАДИ. - № 2015120191/11; заявл. 27.05.2015; опубл. 20.12.15, Бюл. № 35.

4. Щербаков, В.С. Методы управления комплектом машин трубоукладочной колонны : монография / В.С. Щербаков, А. Н. Шабалин, М.С. Корытов. - Омск : СибАДИ, 2014. - 151 с.

5. Щербаков, В.С. Совершенствование системы управления выглаживающей плитой асфальтоукладчика : монография / В.С. Щербаков, С.А. Милюшенко. - Омск : СибАДИ, 2010. $-161 \mathrm{c}$.

6. Щербаков, В.С. Совершенствование системы управления рабочим органом цепного траншейного экскаватора : монография / В.С. Щербаков, Р.Ю. Сухарев. - Омск: СибАДИ, 2011. - 149 c.

7. Щербаков, В.С. Автоматизация проектирования устройств управления положением платформы строительной машины : моногра- фрия / В.С. Щербаков, М.С. Корытов, М.Г. Григорьев. - Омск: СибАДИ, 2011. - 119 с.

8. Щербаков, В.С. Стабилизация несущей платформы в горизонтальной плоскости / В.С. Щербаков, М.С. Корытов, М.Ю. Архипенко, Н.А. Камуз // Строительные и дорожные машины. - 2013. - №2. - С. 36-40.

9. Щербаков В.С. Методика проверки положения грузоподъемного крана в пространстве конфигураций по ограничению на устойчивость / В.С. Щербаков, М.С. Корытов, Н.А. Камуз // Ориентированные фундаментальные и прикладные исследования - основа модернизации и инновационного развития архитектурно-строительного и дорожно-транспортного комплексов России : материалы 66-й Междунар. науч.-практ. конф., 2012 г. / СибАДИ. Омск, 2012. - С. $408-412$.

\section{EFFECT REFERENCE POINT MOUNT COUNTERBALANCING ROPES WAVERING CARGO PIPE- LAYING CRANE}

Abstract. The paper deals with the forced oscillations of the load on the boom-laying crane, fixed by means of the counterweight rope design scheme is reasonable flat-laying crane and made up the differential. 


\section{ТРАНСПОРТНОЕ, ГОРНОЕ И СТРОИТЕЛЬНОЕ МАШИНОСТРОЕНИЕ}

equations of motion-pipelayer crane. The dependence of the maximum deviation of cargo from origin point of attachment of the counterweight rope. Defined rational value balancing rope attachment points for each of the investigated boom.

Keywords: pipe-laying crane, wavering, balancing rope, cargo.

\section{REFERENCES}

1. Sherbakov V.S., Koritov M.S., Kotkin S.V. The automation system simulation jib cranes. Monograph, 2012, 143 p.

2. Sherbakov V.S., Koritov M.S., Postukhova E.I. Automation simulation trajectory of the working body of the robot manipulator. Monograph, 2009, $120 \mathrm{p}$.

3. Sherbakov V.S., Koritov M.S., Tanskiy V.V Pipe-laying crane. Patent RF, no 158094, 2015.

4. Sherbakov V.S., Koritov M.S., Shabalin A. $\mathrm{N}$. A set of management practices of the column pipe-laying machines. Monograph, 2014, $151 \mathrm{p}$.

5. Sherbakov V.S., Milyshenko S.A. Improving governance screed paver. Monograph, 2010, $161 \mathrm{p}$.

6. Sherbakov V.S., Sukharev R.Y. Improving governance working body of the chain trencher. Monograph, 2011, $149 \mathrm{p}$.

7. Sherbakov V.S., Koritov M.S., Grigorev M.G. Design automation controls the position of a construction machine platform. Monograph, 2011, $119 \mathrm{p}$.
8. Sherbakov V.S., Koritov M.S., Arkhipenko M.Y., Kamuz N.A. Stabilization of the support base horizontally. Building and road machines, 2013, no 2, pp. 36-40.

9. Sherbakov V.S., Koritov M.S., Kamuz N.A. Methods of checking the position of the crane in the configuration space to limit the stability. Oriented fundamental and applied research - the basis of modernization and innovative development of architectural-building and roadtransport complex of Russia, Proceedings of the international scientific-practical conference, 2012, no 66, pp. 408-412.

Танский Вячеслав Владимирович (Омск, Россия) - аспирант ФГБОУ ВО “СИбАДИ» (644080, 2. Омск, пр. Мира 5, e-mail: s1ava8968@gmail.com).

Information about the authors

Vyacheslav V. Tanskiy (Omsk, Russian Federation) graduate student of Siberian State Automobile and Highway University "SibADI" (644080, Mira, 5 prospect, Omsk, Russian Federation, e-mail: s1ava8968@gmail.com). 